\title{
Féeries
}

Études sur le conte merveilleux, XVII $-\mathrm{XIX}{ }^{\mathrm{e}}$ siècle

\section{Du gris au doré - La quête d'identité de Cendrillon à travers la couleur}

From Grey to Gold-Cinderella's Self-Discovery in Color

\section{Sabine Schimma}

\section{(2) OpenEdition \\ 1 Journals}

\section{Édition électronique}

URL : https://journals.openedition.org/feeries/3519

DOI : 10.4000/feeries.3519

ISSN : 1957-7753

Éditeur

UGA Éditions/Université Grenoble Alpes

\section{Édition imprimée}

ISBN : 978-2-37747-327-4

ISSN : $1766-2842$

\section{Référence électronique}

Sabine Schimma, «Du gris au doré - La quête d'identité de Cendrillon à travers la couleur », Féeries [En ligne], 17 | 2021, mis en ligne le 17 décembre 2021, consulté le 13 janvier 2023. URL : http:// journals.openedition.org/feeries/3519; DOI : https://doi.org/10.4000/feeries.3519

Ce document a été généré automatiquement le 13 janvier 2023.

Tous droits réservés 


\title{
Du gris au doré - La quête d'identité de Cendrillon à travers la couleur
}

\author{
From Grey to Gold-Cinderella's Self-Discovery in Color
}

\author{
Sabine Schimma
}

1 Le début du XIx ${ }^{e}$ siècle marque l'apogée des travaux de recherche sur les couleurs. Comme jamais auparavant, scientifiques et artistes s'emparent de ce sujet avec complexité et intensité. La couleur est alors libérée de son statut de simple attribut des choses, de qualité sensorielle de second plan, pour gagner son existence propre. Les ouvrages Farbenkugel ( $\mathrm{La}$ sphère des couleurs) du peintre Philipp Otto Runge et Zur Farbenlehre (Traité des couleurs) de Johann Wolfgang von Goethe, intégrant plusieurs disciplines à travers des approches physiques, chimiques mais aussi physiologiques et psychologiques, le prouvent de manière éloquente. Ces deux œuvres sont parues en 1810, peu avant la première édition des Contes de l'enfance et du foyer (en allemand Kinder-und Hausmärchen) écrits par les frères Grimm. Jacob et Wilhelm Grimm avaient connaissance des œuvres de Runge et de Goethe ${ }^{1}$. Ils engagèrent une réflexion sur l'esthétique, sur la portée symbolique des couleurs et leurs effets psychologiques. Dans leur correspondance sur la poésie épique populaire, les frères Grimm se réfèrent en détail à la position de Goethe sur l'esthétique des couleurs. Dans leur dictionnaire de la langue allemande, les concepts de physique, d'effets psychologiques, d'harmonie des couleurs et de matériaux de production des teintures côtoient les définitions du terme « couleur » et de ses composés, des parallèles que fit aussi Goethe ${ }^{2}$.

2 Les Contes de l'enfance et du foyer, contrairement aux contes de fées français de Charles Perrault ou de Marie-Catherine d'Aulnoy parus antérieurement, et contrairement aux contes fantastiques des Romantiques allemands comme Adelbert von Chamisso, ne reflètent pas de discours contemporains mais se distinguent plutôt par leur dimension abstraite et intemporelle. Dans les nombreux remaniements de ces contes, principalement opérés par Wilhelm Grimm, les récits s'ancrent de plus en plus dans un Moyen Âge imaginaire, excluant ainsi toute référence au temps présent ${ }^{3}$. Le caractère novateur résidait dans la fonction attribuée à ces contes, celle de «moyen d'éducation esthétique élémentaire ${ }^{4} »$. 
3 La dimension abstraite évoquée prend tout son sens au $\mathrm{xx}^{\mathrm{e}}$ siècle lorsque Max Lüthi, exégète suisse de renom spécialisé dans les contes, définit le conte populaire comme " un genre très marqué, singulier, montant, se démarquant du caractère complet, profond, nuancé et sentimental de tout ce qui est réel ${ }^{5}$ ». L'auteur établit par ailleurs un lien entre le style de cette forme de narration et le concept d'abstraction de Wilhelm Worringer ${ }^{6}$. Suivant cette approche, le conte est pour Lüthi la métaphore d'un tableau à la surface plane sur laquelle «les personnages se distinguent extérieurement les uns des autres par des contours tranchés et des couleurs pures ${ }^{7}$ ». Les couleurs elles-mêmes ont cette dimension abstraite :

La réalité nous montre une quantité de teintes et d'ombres différentes. Les couleurs mélangées y sont davantage présentes que les couleurs pures. Le conte, au contraire, privilégie la couleur nette et entièrement pure: doré, argenté, rouge, blanc, noir, mais aussi bleu. Le doré et l'argenté ont un éclat métallique, le noir et le blanc sont des contrastes désindividualisés, et le rouge est la couleur la plus marquée. [...] Le gris est la seule couleur mélangée présente et a aussi une dimension métallique dans le conte. [...] Le vert, couleur de la nature vivante, brille par son absence. [...] Les teintes plus subtiles, plus personnelles, comme les camaïeux de brun ou de jaune sont totalement absentes ${ }^{8}$.

4 Les couleurs pures sont bien présentes dans les Contes de l'enfance et du foyer, notamment dans Le Petit Chaperon rouge ou La Lumière bleue. Contrairement à la thèse de Lüthi sur l'abstraction, le gris est aussi présent, il apparaît dans sa valeur symbolique et sans référence directe à son aspect métallisé, en particulier dans les différentes versions du conte de Cendrillon.

5 Si l'approche de Lüthi se concentre uniquement sur le niveau narratif, la désignation explicite des couleurs n'est pas toujours nécessaire dans le conte populaire au niveau de la réception esthétique. Des allusions discrètes, de légers indices ou la simple description vague d'un protagoniste sont autant d'espaces vides que le lecteur peut remplir d'images colorées grâce à son imagination. Les couleurs décrites dans le texte s'affichent ainsi sur un pied d'égalité aux côtés des couleurs et des représentations imaginées. Le conte populaire agit alors comme l'expérience dans l'épistémologie de Goethe: c'est un moyen de médiation entre l'objet et le sujet. Dans le conte, comme chez Goethe, le contenu narratif communiqué est vivant grâce à la participation effective du sujet (qui écoute ou lit l'histoire)'.

6 À la lumière de la théorie des couleurs développée par Goethe, le présent article vise à analyser la version des frères Grimm du conte de Cendrillon (en allemand Aschenputtel) dans lequel le gris et le marron jouent un rôle central. Nous démontrerons que la présence de ces couleurs mélangées dans un conte comme dans un tableau réaliste permet de mettre pleinement en valeur les couleurs pures et de leur donner de la profondeur ${ }^{10}$. L'interprétation qui suit, à la croisée des théories sur les couleurs et sur les contes, vise moins les rapports de causalité logique que la sémantique des couleurs dans le domaine des symboles. Dans cette perspective, les couleurs s'envisagent comme des signes polyvalents dont la connotation varie selon le contexte. Il y a, d'une part, un héritage historique et culturel des couleurs qui entre en jeu et renvoie à des croyances, des usages et des pratiques archaïques et antiques, et, d'autre part, une influence psychologique provenant des couleurs, chaudes ou froides, que Goethe décrit comme «l'effet physico-moral de la couleur ${ }^{11}$ ».

7 Comme l'écrit Gert Theile, le gris connaît une valorisation esthétique autour de 1800. Le programme artistique du Romantisme ne visait pas la précision et la généralisation 
mais l'ambivalence et le caractère diffus des représentations ${ }^{12}$. Cette couleur pâle qu'est le gris a offert aux artistes visuels un grand potentiel de conception. La conscience du potentiel esthétique du gris se manifeste également dans les théories des couleurs développées par Goethe et Runge : selon Goethe, le gris est la somme de toutes les couleurs. Dans sa clarté, cette couleur pâle se situe entre le blanc et le noir, à l'instar des couleurs vives pures, raison pour laquelle Goethe attribue une part d'ombre au gris comme aux couleurs vives. Mais contrairement aux couleurs vives pures, le gris apparaît comme quelque chose de difficilement saisissable, d'indistinct. Un avis que partage également Runge, peintre et spécialiste des couleurs, pour qui le gris est « la cessation de toute individualité ${ }^{13}$ ». Il le place ainsi au centre de son concept de sphère des couleurs de laquelle naissent toutes les autres couleurs. Goethe est certes quelque peu désobligeant en qualifiant le gris de "non-couleur ${ }^{14}$ ", mais il lui offre aussi une place de choix: il la confronte au paradigme dominant de la naissance des couleurs établi par Newton. Ce dernier écrit dans son œuvre Optique que c'est le blanc qui est la somme de toutes les couleurs, tandis que dans la théorie de Goethe, les couleurs physiques naissent de la rencontre entre la lumière et l'obscurité ${ }^{15}$. Cette opacité sombre que représentent les nuances de gris n'est pas seulement un phénomène autonome, c'est aussi un moyen indispensable à la création des couleurs qui marque l'état initial et l'état final de celles-ci ${ }^{16}$. Par cette approche, Goethe serait selon Bernd Hamacher sur la voie d'une symbolique traditionnelle dans laquelle le gris matérialiserait un intervalle existentiel ${ }^{17}$. Celui-ci ne s'inscrit cependant pas dans la durée puisque Goethe attribue au gris un caractère dynamique et changeant.

Dans sa perspective esthétique, Goethe envisage le gris et le marron comme "une opposition entre le froid et le chaud ${ }^{18} »$ : il attribue aux couleurs attirantes, dont le marron fait partie, un effet positif, et aux couleurs repoussantes, dont le gris fait partie, un effet froid et négatif, de sorte que les deux créent ensemble un équilibre. Il conçoit le marron comme une « couleur sale » qui, compte tenu du large spectre de ses tons, se situe entre les couleurs pures et les couleurs pâles, "tantôt modification du jaune, tantôt modification du noir ${ }^{19}$ ».

9 La belle littérature de la période romantique a aussi créé des concepts et des personnages gris et sombres marqués par un potentiel teinté d'indifférence, difficile à cerner et diabolique ${ }^{20}$. Le plus connu de ces personnages est certainement Peter Schlemihl, imaginé par Adelbert von Chamisso, qui vend son ombre à un homme en gris d'apparence insignifiante, mais qui se révèle être le diable. Outre le dictionnaire de la langue allemande des frères Grimm, d'autres œuvres scientifiques analysent le gris et le marron. Leur symbolique renvoie ici et là à un statut esthétique et social modeste. Dans le Traité des couleurs de Goethe, le gris est étroitement lié à l'ombre et, comme pour le marron, la notion de "couleur sale ${ }^{21}$ » est évoquée. Quant au marron, il est mis en parallèle avec la couleur de peau d'une jeune fille qui, contrainte de travailler à la campagne pour assurer sa subsistance, voit sa peau brunie par le soleil22.

Analysons maintenant de plus près le conte de Cendrillon, l'un des favoris des frères Grimm. Le récit observe certes les codes du conte de fée mais, comme toutes les versions du conte, il renferme une grande part de réalisme qui permet une identification au-delà des cercles culturels ${ }^{23}$ et sert d'exemple social. Il était une fois l'ascension sociale d'une jeune fille vertueuse et appliquée qui, après la mort de sa mère, se voit contrainte par sa belle-mère et ses demi-sœurs d'exécuter les pires tâches ménagères et de vivre auprès des cendres du foyer. Des pouvoirs magiques et une robe 
inestimable lui permettront pourtant de conquérir le cœur d'un prince et de se libérer de sa condition de misère. Sujet déjà présent dans de nombreuses œuvres, dont la plus ancienne version en Europe est celle de "La Chatte des cendres » (en italien La gatta cenerentola) parue dans Pentamerone de Giambattista Basile (1634-1636), il sera repris et adapté au XVII ${ }^{\mathrm{e}}$ siècle, comme l'on sait, dans le conte de Cendrillon de Charles Perrault ${ }^{24}$.

11 Les tâches ménagères et la vie auprès du foyer sont synonymes de travaux ingrats et difficiles dans l'Europe du Moyen Âge et du début de l'ère moderne. Cendrillon doit se lever à l'aube, aller chercher de l'eau, faire le feu, la cuisine et la lessive, mais aussi trier des pois et des lentilles renversés dans les cendres par sa belle-mère ${ }^{25}$. Ses demi-sœurs l'enferment dans le rôle de la servante et remplacent ses belles robes par une blouse grise et des sabots en bois. Aux cendres gris-brun se mêlent alors le gris du vêtement et la couleur sans doute brunâtre des souliers. Cendrillon mène ainsi une existence dans l'ombre de la famille, recluse dans la cuisine au fin fond de la maison et enfermée dans la couleur de ses vêtements. Tandis que dans l'œuvre de Perrault, Cendrillon a le droit de dormir au grenier sur une vieille paillasse et reste apparemment d'elle-même auprès $\mathrm{du}$ foyer plein de cendres ${ }^{26}$ (on peut se demander pourquoi), le personnage des frères Grimm vit et travaille uniquement autour du foyer sale, où dominent le gris et le marron. Aucun lieu autre que la cuisine ne lui fait office de chambre.

Comme dans la théorie des couleurs de Goethe, le gris explicitement nommé et le marron imaginé possèdent ici une sémantique polyvalente voire contradictoire, où le foyer est à la fois une sorte de prison et un lieu éphémère. Tout d'abord, dans l'étymologie populaire grecque, la référence aux cendres dans les différentes adaptations du nom du personnage implique une connotation sexuelle suggérant la future relation avec le prince. Les noms font référence à une femme qui se tient auprès du feu, et plus précisément à un chat assis dans les cendres du foyer dont la moitié inférieure du corps est sale ${ }^{27}$. Par ailleurs, les travaux approfondis de recherche psychanalytique mettent en évidence le rétablissement de l'ordre des choses et comparent les tâches de Cendrillon à celles des vestales. Ces prêtresses vierges dédiées à la déesse romaine Vesta étaient chargées d'alimenter le feu et de ramener l'eau d'une source sacrée pour nettoyer le sanctuaire ${ }^{28}$. Protectrice de la maison et du foyer, Vesta est vénérée dans des temples où le feu ne doit jamais s'éteindre pour le salut de l'État tout entier. Enfin, la cendre est elle-même un symbole de purification : comme l'écrit Hedwig von Beit, elle est un hommage au caractère éphémère de la terre dans la tradition antique et chrétienne ${ }^{29}$. La tristesse liée au passé ou à la mort est indispensable pour une renaissance remplie d'espoir. Dans l'Antiquité déjà, mourir par les flammes était symbole de rédemption, comme dans le mythe du phénix qui se jette dans le feu pour renaître de ses cendres, plus jeune et plus puissant. Dans le cas de Cendrillon, c'est la tristesse provoquée par la mort de sa mère qui sous la forme d'un oiseau blanc lui permet d'accéder par magie à une vie nouvelle et heureuse. D'après Eugen Drewermann, le tri des pois et des lentilles (de couleur marron) dans les cendres préfigure déjà ce nouveau départ positif qui a germé dans ce qui n'est plus ${ }^{30}$.

Vers 1800 , le symbole du foyer et de ses cendres, jusqu'alors reflet de la modeste condition sociale de la femme, devient le lieu intermédiaire entre la tombe de la mère de Cendrillon située dans la nature libre et lumineuse, et l'éblouissante salle de bal du château. Par trois fois, Cendrillon s'échappe de la sombre cuisine pour rejoindre la tombe et recevoir de l'arbre magique une magnifique robe. Par trois fois, elle revient dans les cendres après ses escapades interdites dans les terres royales, pour conserver 
l'image de sa modeste condition sociale. Les cendres gris-brun et la cuisine servent ainsi de transition entre un monde tantôt naturel, tantôt artificiel.

Les traces de cendre que Cendrillon a sur le visage et les mains, ce gris-brun qui se matérialise sur le corps et les vêtements du personnage, ouvrent alors une nouvelle perspective symbolique. Cette couche de cendre sur la peau, que Ruth B. Bottigheimer définit comme une métaphore de l'humiliation ${ }^{31}$, rend, en effet, la jeune fille invisible et secondaire dans le cercle familial. La blouse grise et les sabots de bois marron renvoient au statut social modeste de Cendrillon qui a hérité son nom de sa saleté apparente. Derrière les couleurs de ses vêtements se cachent des influences historiques et culturelles : du Moyen Âge au xix ${ }^{\mathrm{e}}$ siècle, le gris et le marron étaient les couleurs des pauvres. Conçus comme des règlementations de l'ordre social, des codes sur les couleurs des vêtements virent le jour, notamment dans les lois somptuaires de Charlemagne en 808. On imposa ainsi le gris et le marron aux personnes des classes modestes, comme les paysans et les artisans. Le choix de ces couleurs n'était pas seulement lié à des raisons de pouvoir et de politique, il était aussi pratique du point de vue économique car portés sans être teintés, les vêtements gris et marron étaient moins chers à la fabrication. Par ailleurs, ces étoffes sombres se salissaient moins rapidement. Les occupants des orphelinats, des hospices et des prisons portaient aussi du gris. Ce concept de simple habit gris donna aussi son nom aux grisettes françaises, ces femmes du XIXe siècle dont les faibles revenus de couturière et d'ouvrière de mode ne permettaient pas l'achat de tenues onéreuses. Dans l'ordre chrétien, le gris et le marron sont les couleurs de l'humilité et de la modestie, de l'abnégation face au monde. Couleurs que les pauvres n'ont pas choisies, elles symbolisent la détresse, l'inquiétude et le manque. Elles ne se remarquent pas, elles sont la douleur silencieuse ${ }^{32}$.

Dans le conte des frères Grimm, les demi-sœurs imposent une tenue de misère à Cendrillon qui était pourtant bourgeoise avant la disparition de sa mère. À l'inverse, chez Perrault, la princesse Peau d'Âne se couvre volontairement d'une peau d'animal grise et marron pour cacher ses origines nobles. Le gris symbolise ainsi la privation de pouvoir et l'humiliation, la douleur silencieuse imposée par les demi-sœurs. Il fait partie du code de la pauvreté, en écho à l'Histoire.

La forte dimension abstraite et intemporelle constatée dans le conte des frères Grimm comparé à ceux de ses prédécesseurs italien et français contraste avec la description détaillée de la blouse et des chaussures que porte Cendrillon. Dans La gatta cenerentola ("La Chatte des Cendres »), Giambattista Basile ne fait que suggérer la transformation des vêtements du personnage, « de la soie et de l'or [en] guenilles ${ }^{33}$ ». Perrault ne parle, lui, que de «méchants habits» qu'il oppose aux magnifiques tenues des demi-sœurs ${ }^{34}$. Dans leurs différentes versions du conte, les frères Grimm insistent de plus en plus sur le contraste entre les habits nobles et la tenue de servante à travers des descriptions toujours plus précises. Dans la première édition des Contes de l'enfance et du foyer (1812), les demi-sœurs "arrachent à Cendrillon sa robe pour l'affubler d'une vieille jupe grise $^{35} »$. Dans la deuxième édition (1819), le contraste esthétique est renforcé et les «beaux habits" sont remplacés par "une vieille blouse grise» vouée aux tâches ménagères ${ }^{36}$. Plus tard, dans leur dernière version de 1857, les sabots de bois font leur apparition $^{37}$. Cette description vestimentaire toujours plus précise renforce le contraste entre le bien et le mal, une opposition qui, comme l'écrivaient eux-mêmes les frères Grimm, « s'exprime souvent par le blanc et le noir, la lumière et l'ombre ${ }^{38}$ ». 
17 Le lieu et les vêtements de travail de Cendrillon sont ainsi intimement liés au gris et au marron, ils oscillent entre clarté et obscurité, entre blanc et noir, comme dans les théories des couleurs de Goethe et de Runge. Pour l'homme d'histoire et de culture qu'est Goethe, le blanc renvoie à la pureté, la simplicité et l'innocence mais porte aussi une connotation liée à la mort et à l'abstrait. Il voit dans le blanc, et sa plus pure manifestation réelle qu'est la neige, la couleur absolue qu'il associe à la lumière et à l'éclat ${ }^{39}$. Ce caractère transcendant se manifeste clairement dans la signification religieuse du blanc qui renvoie à la victoire sur le mal et à l'espoir de résurrection. Citons aussi le Saint-Esprit qui apparaît sous la forme d'une colombe blanche. À l'inverse, le noir est associé à l'obscurité et à la saleté, au mal et à la destruction. Goethe met cette couleur en parallèle avec les ténèbres, avec le plus sombre des phénomènes possibles $^{40}$. Pour les frères Grimm aussi, "l'épouvantable et la noirceur " sont la métaphore du mal qui "s'oppose fortement » aux entités de lumière transcendantes dans le conte ${ }^{41}$. Dans la physique des couleurs de Goethe comme dans les contes « riches en contrastes » des frères Grimm, le blanc (la lumière) doit être réfréné par le sombre pour permettre la naissance des couleurs.

18 Contrairement au noir facilement repérable, le blanc apparaît par touche au fil du conte de Cendrillon. Il accompagne la jeune fille tout au long de sa douloureuse histoire, de sa vie grise et invisible, et symbolise aussi son salut. Après que la neige a recouvert la tombe de la mère et que le soleil a retiré ce "léger voile blanc ${ }^{42}$ ", le père cherche une nouvelle épouse, mère des deux filles qui vont être à l'origine des humiliations de Cendrillon. À la fin du conte, les bas blancs de l'une des demi-sœurs, tâchés du sang rouge de ses talons meurtris, offrent un contraste fort et alertent le prince qui se trompe de fiancée. Le chant de la colombe et la vue du sang convainquent ce dernier de ne pas faire ce mauvais choix. Le prince peut alors rechercher sa vraie promise.

Tout au long du conte, la force transcendante et magique de l'oiseau blanc plane pour aider et sauver Cendrillon de la détresse. Il apparaît seul ou en double à différents endroits. La première fois près du symbolique noisetier où sa blancheur se mêle au vert de la végétation pour créer une alliance féconde : à la demande de sa défunte mère, Cendrillon avait planté sur sa tombe un rameau de noisetier que ses larmes abondantes ont transformé en un arbre magnifique. La dimension métaphorique du flot de larmes et de la pousse rapide de l'arbre reste limitée par le développement naturel du noisetier dont le feuillage vert n'est pas évoqué, mais que le lecteur imagine. Le vert symbolise la croissance et le déploiement de la végétation, ce qui en fait traditionnellement la couleur de l'espoir. Dans la théorie de Goethe, elle est la couleur de l'équilibre psychologique, de la "vraie satisfaction" pour l'œil ${ }^{43}$. De la même façon, elle est connotée chez les frères Grimm dans leur préface de 1815 comme une couleur qui « comble et apaise sans jamais être pesante ${ }^{44}$ ». Pour Cendrillon, le vert symbolise la condition essentielle pour une nouvelle existence. Dans la première édition du conte, la mère mourante exhorte sa fille à la piété et à la bienveillance, et lui promet qu'elle recevra de l'aide directement de l'arbre. À partir de la deuxième édition, l'arbre est indissociable du petit oiseau blanc de qui Cendrillon reçoit ses robes, même si c'est à l'arbre qu'elle s'adresse directement en vers. L'oiseau blanc symbolise l'âme de la mère qui, avant sa mort, en appelle à Dieu et à son aide suprême. À travers lui, elle annonce aussi qu'elle sera toujours auprès de Cendrillon ${ }^{45}$.

Les oiseaux blancs ont une symbolique ambivalente qui apparaît également dans Cendrillon. Outre la symbolique chrétienne du Saint-Esprit proche de la lumière divine 
et annonciateur de la vérité, ils sont aussi l'âme-oiseau dans les croyances populaires et représentent les esprits des défunts. Dans la mythologie antique en revanche, le plumage immaculé et pur des oiseaux blancs en fait les messagers de la déesse de l'amour Aphrodite. Contrairement à la deuxième édition, la première fait apparaître uniquement deux colombes blanches qui aident Cendrillon à trier pois et lentilles des cendres et ont une influence active sur la jeune fille. Elles lui permettent de voir la salle de bal dans le lointain et l'envoient au pied de l'arbre qui lui offrira ses robes. Dans les versions plus tardives, ces deux oiseaux blancs mènent le cortège des autres oiseaux qui aident Cendrillon. La jeune fille va de sa propre initiative auprès de l'arbre et au bal. Dans toutes les versions du conte, les oiseaux blancs endossent le rôle de messagers d'Aphrodite et d'annonciateurs de la vérité en avertissant le prince de l'imposture des fausses fiancées pour qu'il fasse le bon choix.

À partir de la deuxième édition, la connotation positive du blanc associé à la mère de Cendrillon s'oppose au personnage sombre de la belle-mère. Celle-ci l'empêche d'abord de se rendre au bal tant qu'elle n'a pas terminé de trier les pois et les lentilles renversés dans les cendres, pour le lui interdire ensuite, même une fois ce travail accompli. Dans la première édition, ce sont les demi-sœurs qui obligent Cendrillon à exécuter des tâches ingrates. Les frères Grimm renforcent de plus en plus leur caractère obscur. Tandis que la première édition n'évoque qu'un jugement esthétique et moral (les demisœurs "étaient belles en apparence, mais avaient un cœur fier, orgueilleux et malveillant ${ }^{46} »$ ), les frères Grimm accentuent par la suite l'opposition entre l'être et le paraître des deux jeunes filles au moyen de couleurs achromatiques. Elles sont décrites comme "belles et blanches en apparence [...] mais au cœur répugnant et noir ${ }^{47}$ ». Aucun autre passage du conte ne marque aussi bien le contraste entre l'apparence extérieure et le for intérieur d'un personnage. Les vœux des deux jeunes filles viennent aussi appuyer cette contradiction lorsque le père de Cendrillon part en voyage. Elles veulent qu'il leur ramène de belles robes, des perles et des pierres précieuses, autant d'objets qui ne sont qu'artifices, tandis que Cendrillon, socialement rabaissée, ne demande qu'un élément naturel, le premier rameau qui viendra fouetter le chapeau de son père en chemin. De ce même rameau devenu arbre, Cendrillon recevra ensuite à trois reprises des robes à l'éclat authentique, reflet du caractère noble de sa personne.

Dans le conte, le vêtement représente un trait de caractère du personnage ou l'état dans lequel il se trouve. Porteur de sens, il agit comme un moyen de communication non verbale qui cache ou dévoile une vérité, donne des indices sur les problèmes d'identité des personnages ou sert de signe distinctif de leur réelle personnalitét8. Si le gris et le marron masquent la véritable identité positive de Cendrillon, le phénomène d'être et de paraître s'inverse chez ses demi-sœurs. Contrairement à l'analyse de Lüthi, le gris extérieur ne correspond pas à l'état psychique du personnage ${ }^{49}$ mais indique seulement le caractère «trouble » de sa situation temporaire. Si la beauté de Cendrillon perce encore à travers la saleté et les guenilles chez Perrault, la blouse du personnage des frères Grimm cache complètement ses attraits. Ce n'est qu'en se rendant au bal en somptueuse robe dorée et argentée que son moi positif se matérialise dans les nobles étoffes. Cendrillon peut certes profiter de la révélation de sa réelle identité, mais le dévoilement de sa personne l'éloigne paradoxalement des membres de sa famille présents car ils ne la connaissent que vêtue de gris.

Dans les versions allemandes et françaises de Cendrillon et de Peau d'Âne, la transformation des vêtements passant du gris au doré est la démonstration concrète 
que le personnage a mûri et trouvé son identité. Cette évolution établit un parallèle avec le processus alchimique de purification par lequel une matière première noire et obscure est petit à petit transformée en or grâce à la pierre philosophale ${ }^{50}$. Ainsi s'accomplissait la purification spirituelle du fidèle qui atteignait une conscience supérieure grâce à un cœur pur.

Dans les mythes et les contes, la couleur métallisée dorée se situe symboliquement audessus de toutes les autres. Elle renvoie à quelque chose de particulier, d'exceptionnel, qui n'est pas ordinaire. Son rayonnement rappelle la lumière du soleil et des étoiles. Le doré est un feu céleste qui tombe sur terre, le signe du divin dans la tradition chrétienne. Du point de vue de cette sémantique, la rare occurrence naturelle de ce métal noble et sa résistance sont déterminantes. Son caractère précieux en fait le métal des rois ${ }^{51}$. Dans Cendrillon, le doré symbolise une personnalité noble sur laquelle les frères Grimm porte un jugement esthétique : « Tout ce qui est beau est couvert d'or et de perles $[. . .]^{52}$.» Goethe parle aussi de l'effet transcendant de l'or dans ses études sur les couleurs. Il place le doré aux côtés du jaune qu'il compte parmi les couleurs dynamiques et chaudes puisqu'elles créent une atmosphère positive. Pour lui, c'est aussi la dimension transcendantale et éclatante qui distingue le doré du jaune. Souvent cité dans le conte aux côtés du doré, l'argenté est une couleur que Goethe associe au blanc par sa qualité lumineuse encore plus claire ${ }^{53}$. Pour résumer, le blanc et le jaune ainsi que l'argenté et le doré sont synonymes de lumière et, par métaphore, de l'expression de la vérité.

Le dynamisme de l'or fait écho au personnage de Cendrillon qui s'active dans la version des frères Grimm et va se procurer les somptueuses robes sur la tombe de sa mère. Dans la version de Perrault, c'est la fée marraine, bienfaitrice aux pouvoirs magiques, qui habille la jeune fille de la tête aux pieds en transformant ses vieilles guenilles en étoffes neuves et élégantes. Dans la version des frères Grimm, Cendrillon obtient ses magnifiques robes en échange de ses vieux habits. Dans la première édition de 1812, les attributs somptueux font l'objet d'une gradation: lors du premier bal, la jeune fille aperçoit seulement de loin la salle de bal et est éblouie pas tant de splendeurs. Pour le deuxième bal, elle porte une robe argentée et arrive dans un carrosse bleu et argenté. Enfin, lors du troisième bal, elle se rend au château en robe dorée couverte de pierres précieuses et accompagnée de serviteurs en rouge et or, qui représentent pour Goethe les plus nobles des couleurs. Si Cendrillon ne fait qu'entrevoir la magnificence du bal la première fois, c'est son apparition même qui resplendit et illumine le troisième bal. Sa beauté « brille de mille feux » lorsqu'elle entre dans la salle. À son départ de la fête, ses demi-sœurs présentes déclarent: "C'était comme si toutes les lumières s'étaient éteintes d'un coup. » Un vrai contraste s'impose entre l'illumination de la salle de bal et la «petite lampe à huile terne » qui éclaire le modeste logis où Cendrillon se réfugie après chaque apparition ${ }^{54}$.

À compter de la deuxième édition de 1819, Cendrillon n'apparait plus deux fois mais trois fois au bal. La première robe qu'elle porte est déjà parée d'or et d'argent, et sa splendeur et sa magnificence ne cessent de croître. Symbole de la prudence dans les croyances populaires, la couleur argentée apparaît directement à côté du doré, symbole du dynamisme qui rend Cendrillon sûre d'elle pour aller au bal. La jeune fille sait rester modeste mais ne cache pas son enthousiasme pour conquérir le prince. Cette version comporte de nombreuses références à la blouse grise que Cendrillon revêt toujours après avoir retiré ses habits de bal. 
27 La gradation dans l'intensité de l'argenté et du doré s'applique ensuite aux chaussures. Lors du premier bal, la jeune fille reçoit des pantoufles cousues de soie et de fil d'argent. Dans la dernière version des frères Grimm de 1857, Cendrillon échange ses sabots de bois contre des chaussures dorées pour se rendre au troisième bal. Ce contraste esthétique et symbolique atteint son apogée quand la pantoufle dorée reste collée à la poix noire qui recouvre les marches du château. Plus qu'aucun autre passage dans le conte, ce moment révèle la maturité acquise par Cendrillon, délaissant l'ombre et l'obscurité pour une existence dans la lumière. Les marches représentent métaphoriquement la passerelle qui mène au dernier acte de l'histoire - la séance d'essayage de la chaussure ordonnée par le prince pour retrouver sa promise - et conduit Cendrillon vers une vie heureuse. La référence aux croyances populaires germaniques est par ailleurs bien présente : retirer l'ancienne chaussure et enfiler la nouvelle symbolisent la perte de la virginité et le passage à un nouveau statut familial ${ }^{55}$. Les rapports sont désormais inversés en matière de visibilité et d'invisibilité : les demisœurs, qui à la demande de leur mère se sont mutilé les pieds, essayent la chaussure à l'abri du regard du prince pour cacher leur tricherie. Cette notion de cécité présage la suite de l'histoire, quand les colombes s'attaquent aux demi-sœurs lors du mariage de Cendrillon et du prince, et les rendent aveugles. Cette variante du conte sera ajoutée à partir de la deuxième édition. Par deux fois, la belle-mère et le père tenteront aussi d'empêcher Cendrillon d'essayer la chaussure dorée, l'une en prétextant la saleté de la jeune fille, l'autre en déplorant son apparence insignifiante de «petite bête de Cendrillon ${ }^{56}$ ». Après s'être nettoyé le visage et les mains, la jeune fille enfile toutefois la chaussure devant les yeux du prince. Elle enlève son lourd sabot de bois et, par là même, retire le fardeau qu'elle portait dans sa vie, pour se glisser dans la pantoufle dorée et ainsi trouver sa place auprès du prince. La colombe sur l'arbre lumineux qui incarnait jusqu'alors le personnage de la mère défunte se réincarne dans l'éclat des dorures du château.

Nous venons de montrer en quoi la symbolique des couleurs joue un rôle dans la quête d'identité de Cendrillon, et comment le gris et le marron agissent sur le déroulement de l'action du conte, qu'ils soient précisément décrits ou suggérés. Ces deux couleurs interviennent de manière saisissante dans la recherche active d'identité de la jeune fille dont la pauvreté n'est qu'apparente. Comparée à de nombreuses versions du conte de Cendrillon, la signification des couleurs est mise en évidence d'une autre manière dans le conte des frères Grimm, et les éléments liés au toucher ne sont pas valorisés. Alors que d'un point de vue épistémologique l'empirisme du XVII siècle considère la forme, qui correspond au toucher, comme qualité primaire de la connaissance face à la couleur dont la portée n'est qu'ajout et qualité secondaire, la couleur a su s'imposer dans le conte de Cendrillon des frères Grimm. Toutes les actions liées au sens du toucher se déroulent en effet violemment, se terminent sans succès ou nécessitent le sens de la vue pour leur aboutissement. Les exemples suivants sont révélateurs : apparaissant à partir de la deuxième édition, le rameau de noisetier que Cendrillon demande à son père et qu'il semble oublier de lui ramener fouette et fait tomber le chapeau du père comme pour le lui rappeler; lors du premier et du deuxième bal, le prince tient Cendrillon par la main et ne la lâche pas afin qu'elle ne danse avec personne d'autre, pourtant elle lui échappe; la destruction du colombier et l'abattage du poirier dans lequel Cendrillon se cache après le bal ne permettent pas de la retrouver; lors du troisième bal, la pantoufle dorée de la jeune fille reste collée à la poix, en dehors de sa 
volonté ; dans la version de Perrault, le serviteur mandaté par le roi pour l'essayage de la chaussure reconnaît de ses yeux la beauté de la jeune fille malgré la saleté et les guenilles, et le contact de la pantoufle de verre à son pied ne fait que confirmer qu'elle est bien la promise $\mathrm{du}$ prince ${ }^{57}$.

Dans la version des frères Grimm, le rapport entre la couleur et la forme s'inverse : le prince est par deux fois trompé par les demi-sœurs, et seul le chant des colombes lui permet de voir le sang dans la chaussure. Lors du troisième essayage, le prince devenu quelque peu méfiant regarde le visage nettoyé de Cendrillon pour enfin reconnaître la belle danseuse de ses propres yeux. Dans ce conte, la couleur a la qualité sensorielle associée au sentiment et au féminin. Elle l'emporte sur la forme, traditionnellement liée au rationnel et au masculin. La couleur va même jusqu'à remplacer la forme. Comme évoqué précédemment, si pour Lüthi le style du conte populaire s'apparente à une "surface plane" clairement structurée en couleurs pures, frôlant ainsi la métaphore du tableau, ce sont justement les tons obscurs de gris et de marron qui offrent au récit de cette Cendrillon "en retrait " sa profondeur et sa consistance, et «mettent en avant» la couleur dorée. Tandis que les éléments purement liés au toucher ont une connotation davantage négative dans ce conte, le gris et le marron, entre clair et obscur, intègrent la notion de forme dans le royaume de la couleur, comme le décrit aussi Goethe dans sa théorie sur la peinture: "Nous affirmerons maintenant, bien que la chose puisse paraître quelque peu étrange, que l'œil ne voit aucune forme, le clair, l'obscur et la couleur constituant ensemble ce qui pour l'organe distingue un objet de l'autre, et les parties de l'objet entre elles ${ }^{58}$. »

\section{NOTES}

1. Voir par exemple la lettre de W. Grimm à J.Grimm du 14 avril 1809, dans H. Rölleke, Briefwechsel der Brüder Jacob und Wilhelm Grimm, Stuttgart, Hirzel, 2001, t. 1.1, p. 108.

2. Ibid., «Sagenkonkordanz: Farben », t. 1.2, p. 121-124 ; J. et W. Grimm (éds), Deutsches Wörterbuch, Leipzig, Hirzel, 1864, t. 3, col. 1321-1331.

3. La dimension abstraite née de ces contes a définitivement fait du conte un genre littéraire à part entière. H. Hühn et S. Matuschek, « Das aufgeklärte Märchen: Eine europäische Erfolgsgattung von Mme d'Aulnoy und Perrault bis zu den Brüdern Grimm ", Fabula, vol. 55, n 1/2, 2014, p. 2-11; ibid., S. Matuschek, «Es war einmal. Das Märchen als gegenwartsorientierte, dynamische Gattung », p. 13-25.

4. Visant initialement à faire connaître la culture populaire à la classe bourgeoise, le conte s'adresse au cours du XIX ${ }^{\mathrm{e}}$ siècle aux enfants de toutes les classes sociales, notamment par le biais de l'école. R. Steinlein, «Kinder-und Volksmärchen - Anmerkungen zu einer Erfolgsgeschichte », dans C. Brinker-von der Heyde, H. Ehrhardt, H.-H. Ewers et A. Inder (éds), Märchen, Mythen und Moderne. $200 J a h r e$ Kinder-und Hausmärchen der Brüder Grimm, Francfort-sur-le-Main, Internationaler Verlag der Wissenschaften, 2015, p. 607. Sauf indication contraire, toutes les traductions de l'allemand sont de Paule Chauvin.

5. M. Lüthi, Märchen, Stuttgart, Metzler, 2004, p. 31.

6. W. Worringer, Abstraktion und Einfühlung. Ein Beitrag zur Stilpsychologie, Munich, R. Piper \& Co., 1959. 
7. M. Lüthi, Das europäische Volksmärchen, Tubingen, Franke, 1992, p. 25.

8. Ibid., p. 28.

9. J. W. Goethe, «Der Versuch als Vermittler von Objekt und Subjekt » [1793], dans J. W. Goethe, Zur Farbenlehre (Travaux préliminaires), Stuttgart, Verlag Freies Geistesleben, 2003, t. 2, p. 119-131. Voir également l'édition française: "La médiation de l'objet et du sujet dans la démarche expérimentale ", dans P.-H. Bideau (éd.) et H. Bideau (trad.), Traité des couleurs, Laboissière-enThelle, Triades, 1980, p. 296-304.

10. Voir à ce sujet les réflexions de Goethe sur la peinture dans J. W. Goethe, Zur Farbenlehre (Partie didactique), éd. citée, t.1, p. 299-309 (Traité des couleurs, éd. fr. citée, p. 284-290) et A. Hebestreit, Die soziale Farbe. Wie Gesellschaft sichtbar wird, Berlin, LIT Verlag, 2007, p. 242-243.

11. Traité des couleurs, éd. fr. citée, titre de la sixième et dernière partie.

12. G. Theile, "Grauzone des Realen: Annäherung an eine romantische Farbnuance ", dans W. Pape (éd.), Die Farben der Romantik. Physik und Physiologie, Kunst und Literatur, Berlin/Boston, De Gruyter, 2014, p. 191.

13. P. O. Runge, Lettre à Goethe du 3 juillet 1806, dans P.O. Runge, Hinterlassene Schriften, Hambourg, Perthes, 1840, t. 1, p. 97.

14. J. W. Goethe, "Versuch, die Elemente der Farbenlehre zu entdecken», dans J. W. Goethe, Zur Farbenlehre (Travaux préliminaires), éd. citée, t. 2, p. 83.

15. Par moyen opaque, Goethe entend les phénomènes naturels comme l'eau de mer ( $\$ 164)$ et la brume atmosphérique ( $\$ 153-158)$, mais aussi les instruments de physique comme les lentilles et les prismes (§ 178-356, surtout $\S 193,291,292,301,308$ et 318). J. W. Goethe, Zur Farbenlehre (Partie didactique), éd. citée, t. 1, p.107-147. Voir également l'édition française: Traité des couleurs, éd. citée, p. 133-171.

16. B. Hamacher, "Grau und Braun - Vorgefühl der Gegensätze des Kalten und Warmen: Zur Rehabilitierung der farblosen, schmutzigen Farben bei Goethe », dans W. Pape (éd.), ouvr. cité, p. 76.

17. Ibid., p. 75.

18. J. W. Goethe, "Ästhetischer Gebrauch der Farbe ", dans R. Matthaei et al. (éds), Goethe. Die Schriften zur Naturwissenschaft, t. 3.1 : «Beiträge zur Optik und Anfänge der Farbenlehre 1790-1808 », Weimar, Böhlau, 1951, p. 356.

19. Citation de J. W. Goethe, dans P. Schmidt, Goethes Farbsymbolik. Untersuchungen zur Verwendung und Bedeutung der in den Dichtungen und Schriften Goethes, Berlin, Erich Schmidt Verlag, 1965, p. 159.

20. G. Theile, art. cité, p. 193.

21. J. et W. Grimm (éds), Deutsches Wörterbuch, définition de " gris ", t. 8, col. 2073. Disponible sur <https://woerterbuchnetz.de/?sigle=DWB\#1> [consulté le 25/02/2021].

22. Ibid., définition de «marron», t. 2, col.324. Disponible sur <https://woerterbuchnetz.de/? sigle=DWB\#2> [consulté le 25/02/2021].

23. R. Wehse, «Cinderella», dans K. Ranke (éd.), Enzyklopädie des Märchens, Berlin, De Gruyter, 1981, t. 3, p. 39.

24. Ibid., p. 43.

25. J. et W. Grimm, "Aschenputtel », dans J. et W. Grimm, Kinder- und Hausmärchen (Contes de l'enfance et du foyer) [1857], Munich, Winkler, 1977, t. 1, p. 154. Voir également l'édition française qui correspond à la dernière version de 1857 : J. et W. Grimm, «Cendrillon », dans J. et W. Grimm, Contes, trad. M. Robert, Paris, Gallimard, coll. « Folio Classique », 1976, p. 97. Nous renverrons à cette traduction uniquement pour le dernier état du texte, les traductions des autres éditions sont de Paule Chauvin.

26. C. Perrault, «Cendrillon ou la petite pantoufle de verre » [1697], dans C. Perrault, Contes, éd.

T. Gheeraert, Paris, Honoré Champion, 2012, p. 224.

27. R. Wehse, ouvr. cité, p. 43. 
28. Voir B. Bettelheim, Kinder brauchen Märchen, Munich, Deutscher Taschenbuch Verlag, 1990, p. 297 et C. Altmann-Glaser, " Aschenputtel aus Jungscher Sicht », dans Märchenspiegel, Zeitschrift für internationale Märchenforschung und Märchenpflege, $\mathrm{n}^{\circ}$ 3, 2011, p. 23.

29. H. von Beit, Symbolik des Märchens, Berne, A. Francke AG Verlag, 1960, p. 504-505.

30. E. Drewermann, Vom Weg der Liebe. Aschenputtel, Schneewittchen und Marienkind tiefenpsychologisch gedeutet, Ostfildern, Patmos, 2011, p. 66.

31. R. B. Bottigheimer, Schuld und Chance. Die Wertewelt der Grimmschen Märchen, Kromsdorf, Jonas Verlag, 2019, p. 41 [Grimm's Bad Girls \& Bold Boys. The Moral and Social Vision of the Tales, New Haven (Conn.), Yale University Press, 1987].

32. E. Heller, Wie Farben wirken. Farbpsychologie, Farbsymbolik, Kreative Farbgestaltung, Reinbek bei Hamburg, Rowohlt Taschenbuch, 1999, p. 218 et 229-231. Voir également l'édition française : E. Heller, Psychologie de la couleur. Effets et symboliques, Paris, Pyramyd, 2009, p. 217 et 235. Voir enfin P. Thurn, Soziologie der Farbe, Cologne, DuMont Buchverlag, 2007, p. 176-177 ; S. Schimma et P. Stohler (éds), Rotes Käppchen. Blauer Bart. Farben im Märchen/Red Hood. Blue Beard. Colour in Fairy Tales, Kassel, Grimmwelt Kassel gGmbh and Stuttgart, arnoldsche Art Publishers, 2019, p. 92-97.

33. G. Basile, "La Chatte des Cendres », dans Le Conte des contes, trad. F. Decroisette, Paris, Circé, p. 79.

34. C. Perrault, éd. citée, p. 224.

35. J. et W. Grimm, « Aschenputtel », dans K. Derungs (éd.), Die ursprünglichen Märchen der Brüder Grimm. Die wahren Geschichten neu entdeckt, Grenchen bei Solothurn, Amalia, 2010, p. 100.

36. J. et W. Grimm, "Aschenputtel », dans J. et W. Grimm, Kinder- und Hausmärchen (Contes de l'enfance et du foyer), Berlin, Verlag Reimer, 1819, t. 1, p. 115.

37. J. et W. Grimm, "Aschenputtel ", dans J. et W. Grimm, Kinder- und Hausmärchen (Contes de l'enfance et du foyer) [1857], éd. citée, p. 154. Voir également l'édition française : «Cendrillon », éd. citée, p. 97.

38. J. et W. Grimm, «Einleitung. Ueber das Wesen der Märchen », dans J. et W. Grimm, Kinder- und Hausmärchen (Contes de l'enfance et du foyer) [1819], éd. citée, t. 1, p. XXXII.

39. J.W. Goethe, "Versuch, die Elemente der Farbenlehre zu entdecken", dans Zur Farbenlehre (Travaux préliminaires), éd. citée, t. 2, p. 76-77 et ibid. (Partie didactique), éd. citée, t. 1, p. 224. Voir également l'édition française : Traité des couleurs, éd. citée, p. 223.

40. J. W. Goethe, "Versuch, die Elemente der Farbenlehre zu entdecken», dans J. W. Goethe, Zur Farbenlehre (Travaux préliminaires), éd. citée, t. 2, p. 79 et E. Heller, ouvr. cité, p. 93-94.

41. J. et W. Grimm, « Vorrede », dans J. et W. Grimm, Kinder- und Hausmärchen (Contes de l'enfance et $d u$ foyer), Berlin, Realschulbuchhandlung, 1812, t. 1, p. 11.

42. J. et W. Grimm, "Aschenputtel », dans J. et W. Grimm, Kinder- und Hausmärchen (Contes de l'enfance et du foyer) [1857], éd. citée, p. 154 (trad. P. Chauvin, l'édition française citée donne pour traduction « tapis blanc », ce qui est beaucoup moins satisfaisant).

43. J. W. Goethe, Zur Farbenlehre (Partie didactique), éd. citée, t. 1, p. 284. Voir également l'édition française : Traité des couleurs, éd. citée, p. 272.

44. J. et W. Grimm, "Vorrede », dans J. et W. Grimm, Kinder- und Hausmärchen (Contes de l'enfance et du foyer) [1815], éd. citée, t. 2, p. VI.

45. E. Drewermann, ouvr. cité, p. 78 et H. Wöller, Aschenputtel. Energie der Liebe, Zurich, KreuzVerlag, 1984, p. 61.

46. J. et W. Grimm, « Aschenputtel », dans K. Derungs (éd.), ouvr. cité, p. 100.

47. J. et W. Grimm, "Aschenputtel», dans J. et W. Grimm, Kinder- und Hausmärchen (Contes de l'enfance et du foyer), éd. citée, 1819, t. 1, p. 114.

48. K. Horn, «Das Kleid als Ausdruck der Persönlichkeit. Ein Beitrag zum Identitätsproblem im Volksmärchen », dans Fabula, vol. 18, 1977, p. 82-84 et K. Horn, « Kleidung », dans K. Ranke (éd.), ouvr. cité, 1993, t. 7, p. 1432-1441.

49. M. Lüthi, Das europäische Volksmärchen, Tubingen, Franke, 1992, p. 30. 
50. <https://fr.wikipedia.org/wiki/Pierre_philosophale> [consulté le 25/02/2021].

51. E. Heller, ouvr. cité, p. 187. Voir également S. Schimma et P. Stohler (éds), ouvr. cité, p. 82-91.

52. J. et W. Grimm, « Vorrede », dans J. et W. Grimm, Kinder- und Hausmärchen (Contes de l'enfance et du foyer), éd. citée, 1812, t. 1, p. 11.

53. J. W. Goethe, Zur Farbenlehre (Partie didactique), éd. citée, t. 1, p. 208 et 277. Voir également l'édition française : Traité des couleurs, éd. citée, p. 211 et 268.

54. J. et W. Grimm, « Aschenputtel », dans K. Derungs (éd.), ouvr. cité, p. 100.

55. H.-J. Uther, "Aschenputtel», dans H.-J. Uther, Handbuch zu den Kinder- und Hausmärchen der Brüder Grimm. Entstehung, Wirkung, Interpretation, Berlin/Boston, De Gruyter, 2013, p. 54. Voir aussi l'interprétation de Bruno Bettelheim pour qui la pantoufle symbolise le vagin d'un point de vue psychanalytique : B. Bettelheim, ouvr. cité, p. 314.

56. J. et W. Grimm, "Aschenputtel », dans J. et W. Grimm, Kinder- und Hausmärchen (Contes de l'enfance et du foyer) [1857], éd. citée, p. 161. Voir également l'édition française : « Cendrillon », éd. citée, p. 105.

57. Dans le mythe antique de la courtisane Rhodope retranscrit par Strabon, celle-ci se fait dérober une chaussure par un aigle qui la laisse tomber aux pieds d'un pharaon. Sous le charme, il fait rechercher sa propriétaire dans tout le royaume et la séance d'essayage suffit aussi à la retrouver : B. Bettelheim, ouvr. cité, p. 314.

58. J. W. Goethe, Traité des couleurs, éd. citée, p. 88. Voir également l'édition allemande: Zur Farbenlehre (Partie didactique), éd. citée, t. 1, p. 56.

\section{RÉSUMÉS}

Le présent article traite de la symbolique des couleurs pour illustrer le cheminement existentiel de Cendrillon qui passe de l'ombre à la lumière. Paru à la même époque que les Contes de l'enfance et $d u$ foyer des frères Grimm, le Traité des couleurs de Goethe est ici utilisé comme source principale d'interprétation. Si les lieux occupent une place importante dans le conte, en particulier le foyer souillé de cendres, la transformation de la couleur des vêtements reste l'élément essentiel. Cette analyse interroge l'approche de Max Lüthi, exégète suisse spécialisé dans les contes, pour qui les couleurs apparaissent sous leur forme pure afin de construire le style abstrait du conte populaire.

In this text, the symbolism of colors is used to show how Cinderella finds a way to escape from her shadowy existence to live a life of brightness and splendour. The primary interpretation aid is Goethe's Farbenlehre, or Theory of Colours, which was published at the same time as the Brothers Grimm's Children's and Household Tales. Special attention is given not only to the locations in the tale, particularly the dirty, ash-covered stove, but also to the change in the colour of Cinderella's garments. In the context of the analysis, the approach of the Swiss fairy-tale researcher Max Lüthi is addressed, who posits that colours in a folk tale appear in their pure form as an aid to constituting the tale's abstract style. 
INDEX

Keywords : Cinderella (Aschenputtel), Brothers Grimm, Children's and Household Tales, color, Johann Wolfgang von Goethe, Theory of Colours, Max Lüthi, grey, brown, gold

Mots-clés : Cendrillon (Aschenputtel), les frères Grimm, Contes de l'enfance et du foyer, couleur, Johann Wolfgang von Goethe, Traité des couleurs, Max Lüthi, gris, brun, doré

\section{AUTEUR}

SABINE SCHIMMA

Goethe and Schiller Archive in Weimar, Allemagne 